\title{
Severely blunted early heart rate response during treadmill exercise is associated with above average exercise capacity
}

\author{
Afshan Alvi ${ }^{1}$, Ayesha Salahuddin ${ }^{1}$, Cindy Y. Wang ${ }^{1}$, Wilbert S. Aronow ${ }^{2}$, Daniel M. Spevack ${ }^{1}$
}

${ }^{1}$ Division of Cardiology, Montefiore, Albert Einstein College of Medicine, Bronx, NY, USA ${ }^{2}$ Division of Cardiology, Department of Medicine, Westchester Medical Center and New York Medical College, Valhalla, NY, USA

Submitted: 25 May 2019

Accepted: 22 June 2019

Arch Med Sci Atheroscler Dis 2019; 4: e167-e173 DOI: https://doi.org/10.5114/amsad.2019.86758 Copyright @ 2019 Termedia \& Banach

\section{Abstract}

Introduction: Chronotropic response with exercise is evaluated by peak heart rate $(H R)$ achieved. Since most of the exercise-related chronotropic response occurs early after exercise is initiated, we investigated whether the HR achieved with a standard dose of exercise (Bruce stage 2 ) is associated with exercise capacity. We hypothesized that those with a blunted or disproportionate HR response at this exercise dose would have reduced exercise capacity compared to those with a typical HR response.

Material and methods: We reviewed 3,084 consecutive normal maximal treadmill stress echocardiographic reports acquired from individual adults over a 1.5-year period. We examined for association between stage 2 Bruce HR with age and sex-adjusted exercise capacity.

Results: After adjustment for age and sex, Bruce stage 2 HR was inversely associated $(\beta=-0.08, p<0.01)$ with exercise duration. Thus for every additional 10 beats per minute achieved in stage 2 , exercise duration was generally shortened by about $45 \mathrm{~s}$. Most of the subjects (92\%) who had a stage 2 Bruce HR response below the 10th percentile had above average or average exercise capacity for their age and sex.

Conclusions: Lower Bruce stage $2 \mathrm{HR}$ was associated with increased exercise capacity. Severely blunted HR response was associated with above average exercise capacity. Caution should therefore be exercised in attributing exercise intolerance to a blunted HR response when making a diagnosis of chronotropic incompetence.

Key words: chronotropic incompetence, peak heart rate, exercise capacity.

\section{Introduction}

Chronotropic incompetence is usually defined as an inability to increase heart rate $(H R)$ with increased activity or demand [1-3]. The most commonly employed criterion used during clinical stress testing is framed in terms of the peak HR achieved (i.e. $85 \%$ of the maximum predicted heart rate for age (MPHR)) [4]. This definition, however, ignores two key parameters necessary to evaluate the adequacy of the HR response, namely the rate of HR increase and the effort made by a patient in order to raise the $H R$.

The exercise HR response is not a linear relationship. Most of the increase in rate occurs during the first $5 \mathrm{~min}$ of a standard Bruce proto-

\author{
Corresponding author: \\ Prof. Wilbert S. Aronow MD, \\ FACC, FAHA \\ Cardiology Division \\ Westchester Medical \\ Center and New York \\ Medical College \\ Macy Pavilion \\ Room 141 \\ Valhalla, NY 10595, USA \\ Phone: 914-493-5311 \\ Fax: 914-235-6274 \\ E-mail:wsaronow@aol.com
}


col. We aimed to investigate the Bruce stage $2 \mathrm{HR}$ as a parameter of chronotropic response since it both incorporates the rate of HR rise and it allows comparison of individuals after a standard dose of exercise. We hypothesized that those with either blunted or disproportionate HR responses would have reduced exercise capacity compared to those with a typical HR response.

\section{Material and methods}

\section{Participants}

From a query of our institution's cardiology database (Lumedx, Oakland CA) we identified 4,495 consecutive subjects who underwent treadmill stress echocardiography between January 1, 2010 and July 1,2012 . After exclusion for age $<18$ years $(n=12)$, resting HR $>90 \mathrm{bpm}(n=430)$, abnormal resting left ventricle $(\mathrm{LV})$ wall motion $(n=41)$, inability to complete stage 2 of the Bruce Protocol ( $n=697)$, ischemic exercise response $(n=166)$ and repeat testing $(n=65)$, the study cohort consisted of 3,084 individuals.

Clinical data were acquired using our institutional electronic patient information database (Clinical Looking Glass (CLG); Montefiore IT, Yonkers, NY). This database integrates clinical data from all inpatient visits at three facilities and outpatient encounters at twenty ambulatory sites, home care and community service programs with- in the Montefiore system. Information extracted included comorbidities, race/ethnicity and medications prescribed. CLG also captured all dates of death from a National Death Index and from the hospital's inpatient record. Montefiore Medical Center's institutional review board approved the study.

\section{Ethical statement}

Institutional review board approval was obtained for this study.

\section{Statistical analysis}

Statistical analysis was performed using Stata software v11 (College Station, TX). Comparison of means was performed using the two-sample $t$-test. Comparison of categorical data was performed using the $\chi^{2}$ test. $P$-values were considered significant if $p<0.05$.

\section{Results}

Clinical characteristics of the study cohort are displayed in Table I. Male subjects had a higher prevalence of smoking, diabetes and kidney disease (CKD stage > 3). Male subjects were more likely to have been prescribed $\beta$-blockers (all tests were performed with $\beta$-blockers held for more than $24 \mathrm{~h}$ ). Female subjects had slightly higher baseline LV ejection fraction, higher resting HR

Table I. Patient demographics, comorbidities and rest/exercise vital signs stratified by sex

\begin{tabular}{|c|c|c|c|}
\hline Parameter & Males & Females & $P$-value \\
\hline Number of subjects & $1,359(44 \%)$ & $1,725(56 \%)$ & \\
\hline Age [years] & $52 \pm 12$ & $52 \pm 12$ & 0.95 \\
\hline Height $[\mathrm{cm}]$ & $174 \pm 8$ & $161 \pm 7$ & $<0.01$ \\
\hline Weight $[\mathrm{kg}]$ & $88 \pm 17$ & $76 \pm 16$ & $<0.01$ \\
\hline BMI $\left[\mathrm{kg} / \mathrm{m}^{2}\right]$ & $29 \pm 5$ & $29 \pm 6$ & 0.15 \\
\hline Smoker (\%) & 17 & 13 & $<0.01$ \\
\hline Hypertension (\%) & 54 & 52 & 0.40 \\
\hline Diabetes (\%) & 26 & 19 & $<0.01$ \\
\hline Kidney disease (\%) & 12 & 9 & $<0.01$ \\
\hline B-blocker (\%)* & 23 & 18 & $<0.01$ \\
\hline Ejection fraction (\%) & $62 \pm 5$ & $64 \pm 5$ & $<0.01$ \\
\hline Resting HR [bpm] & $68 \pm 10$ & $70 \pm 10$ & $<0.01$ \\
\hline Resting SBP [mm Hg] & $130 \pm 18$ & $125 \pm 19$ & $<0.01$ \\
\hline Stage 2 Bruce HR [bpm] & $127 \pm 19$ & $137 \pm 18$ & $<0.01$ \\
\hline Peak HR [bpm] & $158 \pm 18$ & $157 \pm 20$ & 0.86 \\
\hline Peak SBP [mm Hg] & $171 \pm 25$ & $162 \pm 23$ & $<0.01$ \\
\hline
\end{tabular}

${ }^{*}$ Prescribed $\beta$-blockers were held for $24 \mathrm{~h}$ before stress testing. 
and lower resting systolic blood pressure. They also had higher stage 2 Bruce HR and lower systolic blood pressure at peak exercise than males.

Table II shows the typical HR and exercise duration stratified by sex and age groups. The ranges for the peak and Bruce stage $2 \mathrm{HR}$ displayed in the table show the $10^{\text {th }}$ and $90^{\text {th }}$ percentile values for age and sex. These cutoffs were chosen to exclude those with HR responses that were extremely atypical. Those with Bruce stage $2 \mathrm{HR}$ below the $10^{\text {th }}$ percentile were categorized as having a "blunted" HR response, whereas those above the $90^{\text {th }}$ percentile were categorized as having a "disproportionate" HR response. The ranges displayed in the table for Bruce exercise duration capture the $25^{\text {th }}$ to the $75^{\text {th }}$ percentile for age and sex. These cutoffs were chosen to highlight the most typical of exercise responses seen, since a substantial amount of variability in exercise tolerance was observed even within specific age and sex groups. Those with exercise duration below the $25^{\text {th }}$ percentile were categorized as having "below average" exercise capacity, whereas those above the $75^{\text {th }}$ percentile were categorized as having "above average" exercise capacity. Overall, female subjects had higher Bruce stage 2 HR (137 \pm 18 vs. $128 \pm 18$ bpm, $p<0.01$ ), whereas peak HR achieved was similar for both sexes (158 \pm 20 vs. $158 \pm 18, p=0.8$ ).

Figure 1 shows the exercise HR response stratified by the HR response category. At the end of
Bruce stage 2, the average HR values were 101 $\pm 12,133 \pm 13$ and $165 \pm 10$ bpm (ANOVA $p<0.001$ ) respectively in those with blunted, typical and disproportionate HR responses. Figure 2 shows the relationship between $H R$ response category and exercise duration category. Subjects with blunted $H R$ response tended to have above average exercise capacity compared to the other groups ( $p<$ 0.001 ). Those with a typical HR response tended to have average exercise capacity compared to the other groups $(p<0.001)$. Disproportionate $\mathrm{HR}$ response was associated with below average

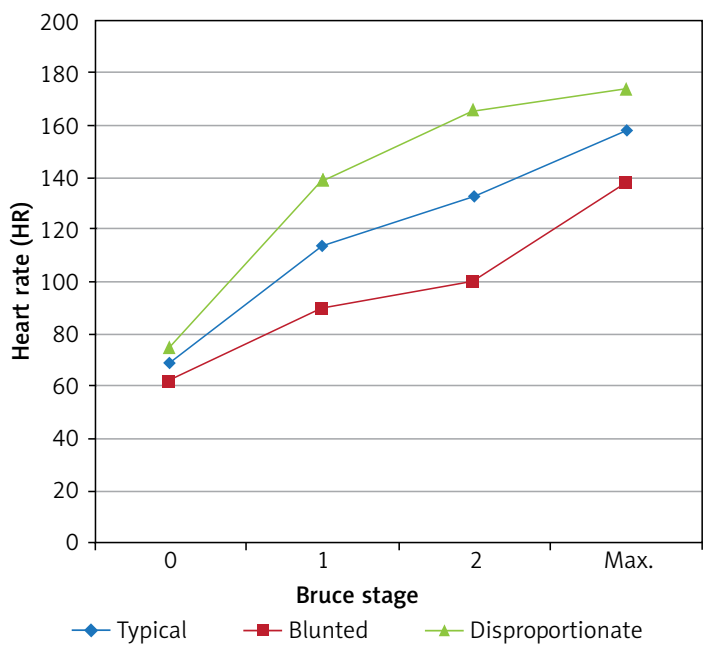

Figure 1 . The exercise heart rate response stratified by the HR response category

Table II. Peak HR (10 th $90^{\text {th }}$ percentile), stage $2 \mathrm{HR}\left(10^{\text {th }}\right.$ to $90^{\text {th }}$ percentile) and exercise duration $\left(25^{\text {th }}\right.$ to $75^{\text {th }}$ percentile). Results are stratified by sex and age groups

\begin{tabular}{|c|c|c|c|c|c|c|c|}
\hline \multicolumn{4}{|c|}{ Males } & \multicolumn{4}{|c|}{ Females } \\
\hline $\begin{array}{l}\text { Age } \\
\text { [years] }\end{array}$ & $\begin{array}{l}\text { Peak HR } \\
\text { [bpm] }\end{array}$ & $\begin{array}{l}\text { Stage } 2 \mathrm{HR} \\
\text { [bpm] }\end{array}$ & $\begin{array}{c}\text { Duration (mm:ss) } \\
-I Q R\end{array}$ & $\begin{array}{c}\text { Age } \\
\text { [years] }\end{array}$ & $\begin{array}{l}\text { Peak HR } \\
\text { [bpm] }\end{array}$ & $\begin{array}{l}\text { Stage } 2 \mathrm{HR} \\
\text { [bpm] }\end{array}$ & $\begin{array}{c}\text { Duration (mm:ss) } \\
-I Q R\end{array}$ \\
\hline$<20$ & $173-200$ & $114-136$ & $12: 00$ to $14: 05$ & $<20$ & $181-196$ & $134-171$ & $9: 00$ to $12: 00$ \\
\hline $21-25$ & $176-188$ & $113-140$ & $10: 11$ to $15: 00$ & $21-25$ & $171-191$ & $126-160$ & $9: 27$ to $11: 20$ \\
\hline $26-30$ & $173-190$ & $120-150$ & $9: 32$ to $12: 54$ & $26-30$ & $171-181$ & $131-155$ & $8: 49$ to $10: 56$ \\
\hline $31-35$ & $169-184$ & $117-141$ & $9: 33$ to $12: 15$ & $31-35$ & $166-184$ & $133-153$ & $7: 45$ to $10: 22$ \\
\hline $36-40$ & $162-179$ & $120-139$ & $9: 18$ to $12: 04$ & $36-40$ & $162-179$ & $133-155$ & $7: 30$ to $10: 41$ \\
\hline $41-45$ & $156-176$ & $118-142$ & $8: 47$ to $12: 00$ & $41-45$ & $155-173$ & $130-153$ & $7: 02$ to $9: 50$ \\
\hline $46-50$ & $152-173$ & $116-139$ & $8: 40$ to $11: 05$ & $46-50$ & $150-171$ & $126-149$ & $6: 51$ to $9: 46$ \\
\hline $51-55$ & $146-171$ & $114-139$ & $8: 09$ to $11: 40$ & $51-55$ & $148-166$ & $125-148$ & $7: 01$ to $9: 15$ \\
\hline $56-60$ & $141-162$ & $113-137$ & $7: 31$ to $10: 31$ & $56-60$ & $144-164$ & $125-148$ & $6: 31$ to $10: 08$ \\
\hline $61-65$ & $139-164$ & $113-141$ & $7: 16$ to $9: 56$ & $61-65$ & $141-162$ & $125-148$ & $6: 13$ to $9: 35$ \\
\hline $66-70$ & $135-156$ & $114-142$ & $6: 46$ to $9: 32$ & $66-70$ & $134-157$ & $120-144$ & $6: 02$ to $8: 10$ \\
\hline $71-75$ & $130-148$ & $116-144$ & $6: 13$ to $8: 40$ & $71-75$ & $131-155$ & $121-150$ & $6: 00$ to $8: 18$ \\
\hline $76-80$ & $127-146$ & $111-134$ & $6: 00$ to $9: 00$ & $76-80$ & $126-139$ & $113-131$ & $6: 10$ to $7: 44$ \\
\hline$>80$ & $121-139$ & $100-139$ & $6: 0(\mathrm{HR}) 0$ to $08: 35$ & $>80$ & $118-129$ & $112-127$ & $6: 00$ to $7: 00$ \\
\hline
\end{tabular}



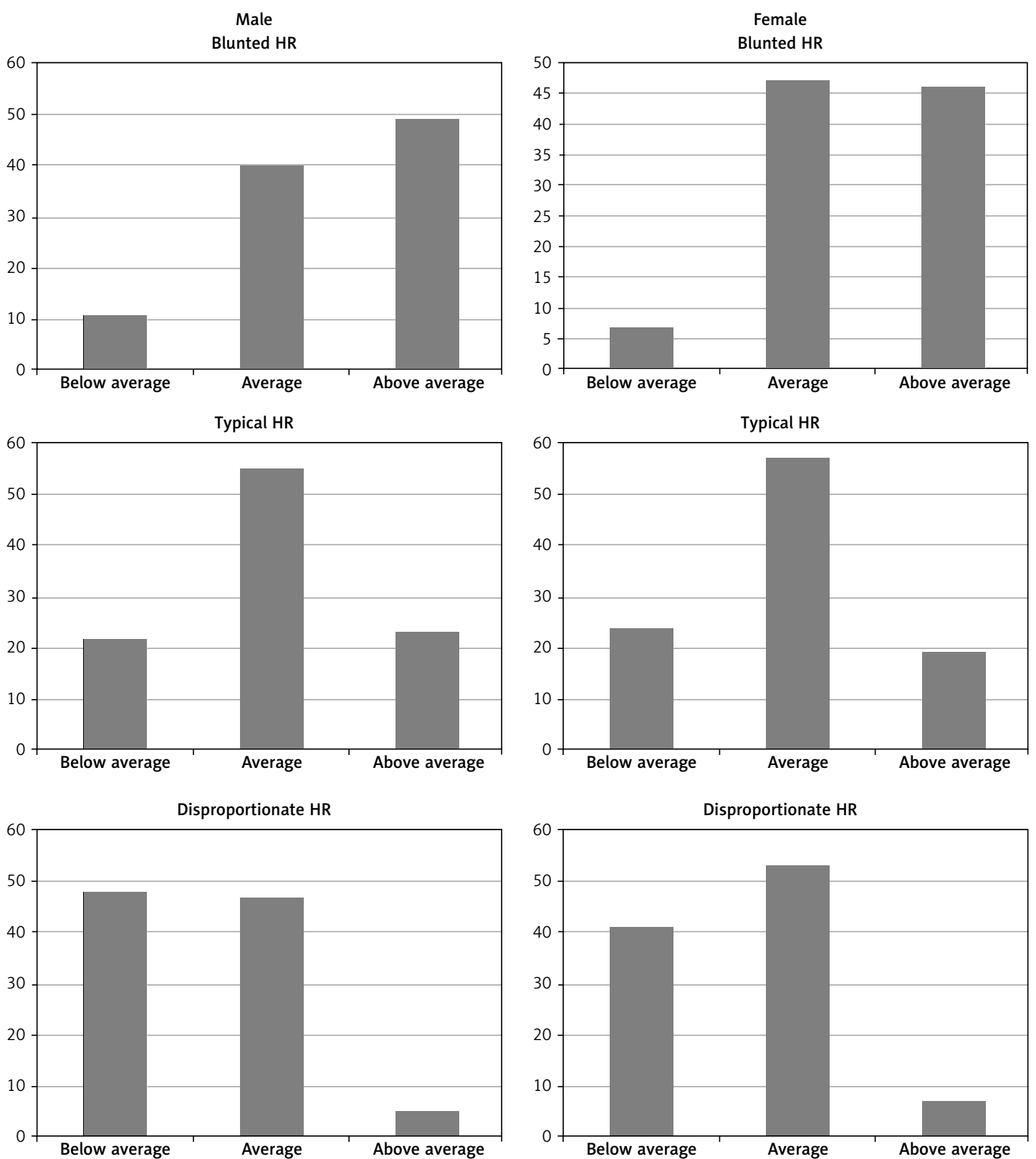

Figure 2. The relationship between HR response category and exercise duration category stratified by sex

exercise capacity compared to the other groups $(p<0.001)$. These associations were similar when stratified by sex (Figure 2) and by age (Figure 3). Ninety-three percent of females and $89 \%$ of males with blunted HR responses had above average or average exercise capacity (Figure 2). Ninety-four percent of those older than 60 years and $90 \%$ of those younger than 60 years who had a blunted HR response had above average or average exercise capacity (Figure 3). Ninety-two percent of subjects with stage 2 Bruce HR under $100 \mathrm{bpm}$ $\left(<5^{\text {th }}\right.$ percentile) had above average or average exercise capacity.

Many prior studies have used the criterion of failure to reach $85 \%$ MPHR (where MPHR is given by 220 -age) to indicate an abnormally slow HR response to exercise. The association of stage 2 Bruce HR and exercise capacity was examined amongst those not reaching $85 \%$ MPHR $(n=352)$. In this group, $82 \%$ of those who had blunted stage 2 Bruce HR achieved above average or average exercise capacity. Of those who did not have blunted stage 2 Bruce HR, only 47\% achieved above average or average exercise capacity.

Multivariate linear regression was used to determine the degree of association between HR achieved and exercise capacity. We found that the Bruce stage $2 \mathrm{HR}$ and the peak HR had opposite effects on the subject's exercise duration. After adjustment for age and sex, Bruce stage $2 \mathrm{HR}$ was inversely associated with exercise duration. Thus for every additional 10 beats per minute 

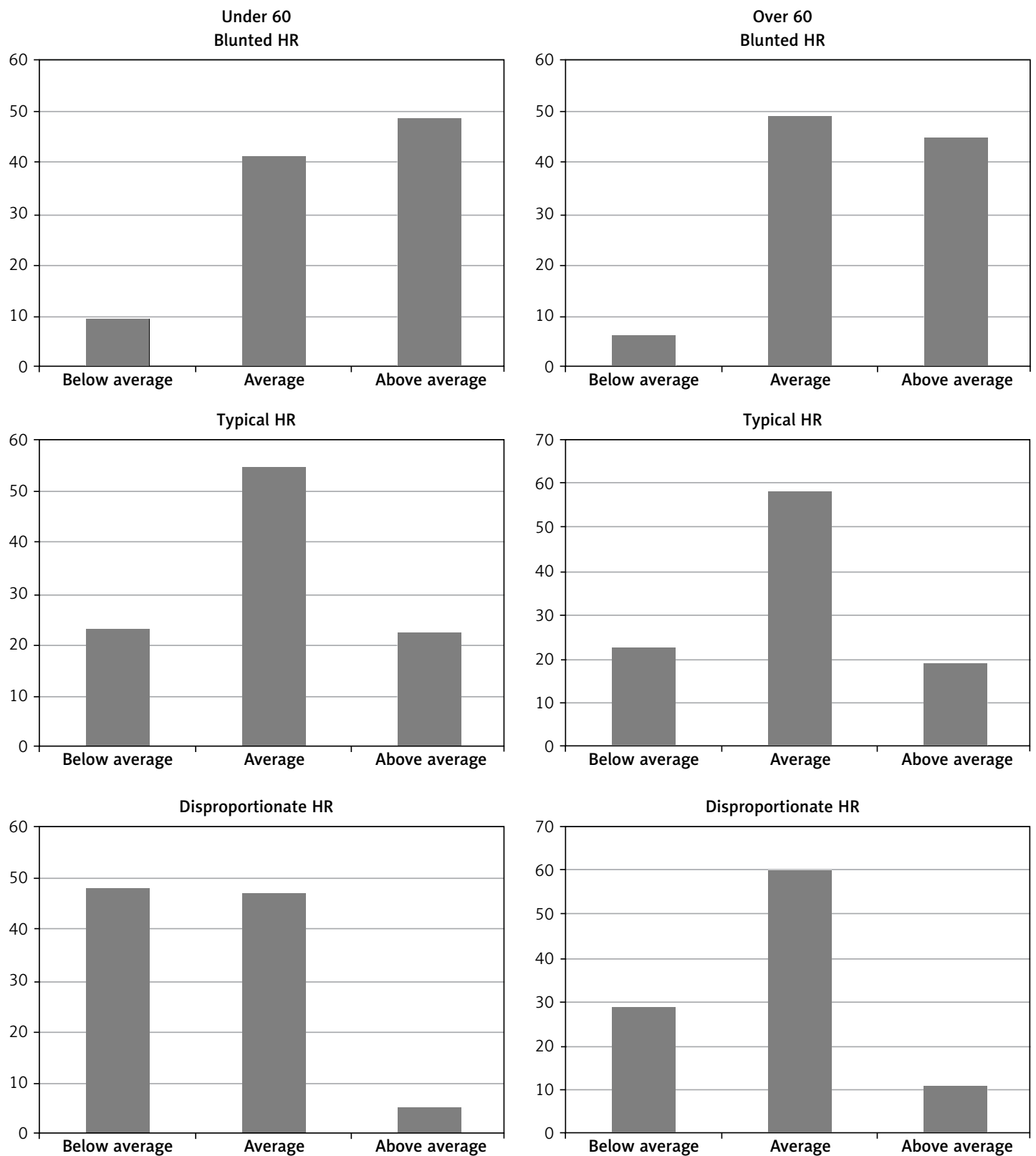

Figure 3. The relationship between HR response category and exercise duration category stratified by age below and over 60 years of age

achieved in stage 2, exercise duration was generally shortened by about $45 \mathrm{~s}$. Conversely, the peak HR achieved had the opposite effect. For every additional 10 beats per minute achieved, exercise duration was generally increased by $45 \mathrm{~s}$. While stage 2 Bruce HR and peak HR were significantly correlated with each other $(p<0.001)$, the correlation was fairly weak $\left(R^{2}=0.24\right)$, indicating just minimal multicollinearity.

Linear regression was also used to examine for an association between HR change occurring both before and after stage 2 Bruce and exercise capacity. HR change from rest to stage 2 Bruce was inversely associated with age/sex adjusted exercise duration $(\beta=-0.03, p<0.01)$. Conversely, the HR change from stage 2 Bruce until peak HR was positively associated with age/sex-adjusted exercise duration $(\beta=0.08, p<0.01)$. When both parameters were included simultaneously in the linear regression model, only the HR change occurring after stage 2 Bruce was associated with age/sex-adjusted exercise duration ( $\beta=0.08, p<$ $0.01)$. Nearly all subjects (97\%) in whom HR increased by more than $36 \mathrm{bpm}$ (top $25^{\text {th }}$ percentile) between stage 2 Bruce and peak exercise had above average or average exercise capacity.

\section{Discussion}

The main finding of this study is that slower exercise HR response (achieved at stage 2 of Bruce 
protocol) is associated with longer exercise duration. Even those at the extreme, with Bruce stage 2 $\mathrm{HR}$ below the $5^{\text {th }}$ percentile, tended to have better than average exercise capacity. This response was true for both sexes and was also true for both younger and older subjects. While we had initially hypothesized that those toward both extremes of HR at Bruce stage 2 would have reduced exercise capacity, our finding is not completely surprising and does have a plausible physiologic basis. Aerobic conditioning seen in athletes is known to increase vagal tone, resulting in lower resting $H R$ [5-8]. Slower rate of HR rise is likely a marker for better aerobic conditioning.

Our finding that reduced peak HR was associated with lower exercise duration is in agreement with multiple prior studies [9-11]. Many authors have interpreted this result to mean that those who do not increase their sinus rates adequately with exercise have chronotropic incompetence, which may be the cause of patient exercise intolerance [12-14]. The results of our study show that the HR response is more complicated than that. Amongst those who did not reach $85 \%$ MPHR for age, exercise capacity tended to be average or above average if the stage 2 Bruce HR was blunted. In contrast, the majority of those without blunted stage $2 \mathrm{HR}$ had below average exercise capacity. Based on these results, it is clear that the peak HR achieved should not be relied on alone to determine whether a patient's chronotropic response is normal. The use of the peak HR achieved as a measure of chronotropic response is also a flawed concept since it does not compare subjects at an equal level of effort. A subject who reaches $85 \%$ MPHR after 3 min of exercise does not have the same chronotropic response as another who requires $15 \mathrm{~min}$ to achieve $85 \% \mathrm{MPHR}$.

The change in HR between stage 2 Bruce and peak exercise also proved to be an important predictor of exercise capacity. This is not surprising, since those with low stage 2 Bruce HR and high peak HR achieved tended to exercise the longest for their age and sex. This relationship has been examined by other researchers [12-14]. In fact, Savonen et al. showed that an attenuated heart rate response in the latter half of exercise rather than initial phases is an independent predictor of mortality [14].

Our study has several important limitations. The study population consisted of subjects referred for clinical stress echocardiograms, generally performed to evaluate signs or symptoms of ischemic heart disease. Those who had an abnormal test at rest or at peak exercise were excluded from this study. We also excluded those who did not complete stage 2 Bruce and those who had abnormal ventricular function. Given these selec- tion criteria, it is plausible that those who had true symptomatic chronotropic incompetence were excluded from our study sample. As previously mentioned, most subjects in our sample who had a blunted HR response (either at stage 2 Bruce or peak exercise) had average or above average exercise capacity. This may indicate that we did not have a large sample with extremely severe sinus node dysfunction. Another limitation is that we used graded exercise testing as our measure of sinus response to exercise. We did not test more sudden onset of exertion like that a patient might encounter when running to catch a bus.

In conclusion, slow HR response at stage 2 of the Bruce protocol was associated with increased exercise capacity. Those with the most severely blunted HR response at that stage tended to have above average exercise capacity for their age and sex. Caution should therefore be exercised in attributing exercise intolerance to a blunted HR response when making a diagnosis of chronotropic incompetence.

\section{Conflict of interest}

The authors declare no conflict of interest.

\section{References}

1. Brubaker PH, Kitzman DW. Chronotropic incompetence: causes, consequences, and management. Circulation 2011; 123: 1010-20.

2. Lauer MS, Okin PM, Larson MG, et al. Impaired heart rate response to graded exercise. Prognostic implications of chronotropic incompetence in the Framingham Heart Study. Circulation 1996; 93: 1520-6.

3. Gulati M, Shaw LJ, Thisted RA, et al. Heart rate response to exercise stress testing in asymptomatic women: the St. James women take heart project. Circulation 2010; 122: $130-7$.

4. Tanaka H, Monahan KD, Seals DR. Age-predicted maximal heart rate revisited. J Am Coll Cardiol 2001; 37: 153-6.

5. Jensen-Urstad K, Saltin B, Ericson M, et al. Pronounced resting bradycardia in male elite runners is associated with high heart rate variability. Scand J Med Sci Sports 1997; 7: 274-8.

6. Uusitalo AL, Laitinen T, Vaisanen SB, et al. Effects of endurance training on heart rate and blood pressure variability. Clin Physiol Funct Imaging 2002; 22: 173-9.

7. Lester M, Sheffield LT, Trammell P, Reeves TJ. The effect of age and athletic training on the maximal heart rate during muscular exercise. Am Heart J 1968; 76: 370-6.

8. Ellestad MH. Chronotropic incompetence. The implications of heart rate response to exercise (compensatory parasympathetic hyperactivity?). Circulation 1996; 93: 1485-7.

9. Christensen NJ, Galbo H. Sympathetic nervous activity during exercise. Annu Rev Physiol 1983; 45: 139-53.

10. Blomqvist CG, Saltin B. Cardiovascular adaptations to physical training. Annu Rev Physiol 1983; 45: 169-89.

11. Fox K, Borer JS, Camm AJ, et al. Resting heart rate in cardiovascular disease. J Am Coll Cardiol 2007; 50: 823-30. 
12. Dresing TJ, Blackstone EH, Pashkow FJ, et al. Usefulness of impaired chronotropic response to exercise as a predictor of mortality, independent of the severity of coronary artery disease. Am J Cardiol 2000; 86: 602-9.

13. Lauer MS, Francis GS, Okin PM, et al. Impaired chronotropic response to exercise stress testing as a predictor of mortality. JAMA 1999; 281: 524-9.

14. Savonen KP, Kiviniemi V, Laukkanen JA, et al. Chronotropic incompetence and mortality in middle-aged men with known or suspected coronary heart disease. Eur Heart J 2008; 29: 1896-902. 